\title{
A LOEWNER APPROACH TO A COEFFICIENT INEQUALITY FOR BOUNDED UNIVALENT FUNCTIONS
}

\author{
DUANE W. DE TEMPLE AND JAMES A. JENKINS ${ }^{1}$
}

\begin{abstract}
The Loewner theory is used to obtain the sharp upper bound for the functional $\operatorname{Re}\left\{e^{2 i \theta}\left(a_{3}-a_{2}^{2}\right)+4 \sigma e^{i \theta} a_{2}\right\}$ over the class of univalent functions $f(z)=b\left(z+a_{2} z^{2}+a_{3} z^{3}+\ldots\right)$ which map the unit disc into itself; $\theta \in \mathbf{R}, \sigma \in[0,1]$, and $b \in(0,1]$ are fixed parameters.
\end{abstract}

Let $S(b), b \in(0,1]$, denote the class of analytic and univalent functions $f(z)=b\left(z+a_{2} z^{2}+a_{3} z^{3}+\ldots\right)$ which map the unit disc $|z|<1$ into itself. Functionals of the coefficients $\left(a_{2}, a_{3}\right)$ can in many cases be estimated by a suitable choice of the parameters $\sigma \in[0,1]$ and $\theta \in \mathbf{R}$ in the sharp inequality

$$
\begin{array}{rlr}
\operatorname{Re}\left\{e^{2 i \theta}\right. & \left.\left(a_{3}-a_{2}^{2}\right)+4 \sigma e^{i \theta} a_{2}\right\} \\
& \leqslant \begin{cases}1-b^{2}-4 \sigma^{2} \log b, & 0<\sigma<b, \\
1+b^{2}-4 \sigma^{2} \log \sigma+6 \sigma^{2}-8 \sigma b, & b<\sigma<1 .\end{cases}
\end{array}
$$

The limiting case $b \rightarrow 0^{+}$yields

$$
\begin{aligned}
& \operatorname{Re}\left\{e^{2 i \theta}\left(a_{3}-a_{2}^{2}\right)+4 \sigma e^{i \theta} a_{2}\right\} \\
& \leqslant 1-4 \sigma^{2} \log \sigma+6 \sigma^{2}, \quad 0 \leqslant \sigma \leqslant 1,
\end{aligned}
$$

an inequality valid for functions $z+a_{2} z^{2}+a_{3} z^{3}+\ldots$ in the class $S$. (The right side is defined by continuity at $\sigma=0$.)

Inequality (1) above was proved originally by variational methods [1]; (2) was obtained as an application of the General Coefficient Theorem [3]. Recently, however, Leeman [4] showed how (2) can be deduced from the Loewner theory. Our purpose here is to considerably simplify Leeman's argument and at the same time derive the more general inequality (1).

It is convenient to replace the $t$ in the usual formulation of the Loewner theory by $x=e^{-t}$, where $b=e^{-t_{0}}$. (See Chapter 6 of Hayman [2], in particular pp. 135-136.) There is then known to exist a dense subclass of functions $f(z)=b\left(z+a_{2} z^{2}+a_{3} z^{3}+\ldots\right)$ in $S(b)$ for which

$$
\begin{gathered}
a_{2}=2 \int_{b}^{1} k(x) d x, \\
a_{3}-a_{2}^{2}=-2 \int_{b}^{1} x k^{2}(x) d x,
\end{gathered}
$$

Received by the editors January 24, 1977.

AMS (MOS) subject classifications (1970). Primary 30A32, 30A34.

Key words and phrases. Univalent functions, bounded univalent functions.

${ }^{1}$ Research supported in part by the National Science Foundation, Grant No. MCS 76-15545. c American Mathematical Society 1977 
where $k(x)=e^{i \alpha(x)}$ is a continuous function on $[b, 1]$ taking values on the unit circle. For this dense subclass we then have

$$
\begin{aligned}
\operatorname{Re}\left(a_{3}-a_{2}^{2}+4 \sigma a_{2}\right) & =2 \operatorname{Re} \int_{b}^{1}\left(4 \sigma k(x)-x k^{2}(x)\right) d x \\
& =2 \int_{b}^{1}(4 \sigma \cos \alpha(x)-x \cos 2 \alpha(x)) d x \\
& =1-b^{2}+4 b \int_{b}^{1}\left(2 \sigma \cos \sigma(x)-x \cos ^{2} \alpha(x)\right) d x .
\end{aligned}
$$

For each $x$ we maximize the integrand over all possible values $t \in[-1,1]$ of $\cos \alpha(x)$. Since the integrand can be written in the form

$$
2 \sigma t-x t^{2}=\frac{\sigma^{2}}{x}-x\left(t-\frac{\sigma}{x}\right)^{2}
$$

it is apparent its maximum value, in the case $\sigma<x$, is $\sigma^{2} / x$, and it occurs at $t=\cos \alpha(x)=\sigma / x$. For $\sigma>x$ the maximum value of the integrand is $2 \sigma-x$, and occurs at $t=\cos \alpha(x)=1$.

Now consider two cases:

Case I. $0 \leqslant \sigma \leqslant b$. The last expression in (3) is bounded above by

$$
1-b^{2}+4 \int_{b}^{1} \frac{\sigma^{2}}{x} d x=1-b^{2}-4 \sigma^{2} \log b .
$$

Case II. $b \leqslant \sigma \leqslant 1$. The last expression in (3) is bounded above by

$$
\begin{aligned}
1-b^{2}+4 \int_{b}^{\sigma}(2 \sigma-x) d x+4 \int_{\sigma}^{1} \frac{\sigma^{2}}{x} d x \\
=1+b^{2}-4 \sigma^{2} \log \sigma+6 \sigma^{2}-8 \sigma b .
\end{aligned}
$$

Replacing $f$ by its rotation $e^{-i \theta} f\left(e^{i \theta} z\right)$ gives the desired inequality. Equality in Case I will hold for the Loewner slit mapping which corresponds to the choice $\alpha(x)=\cos ^{-1}(\sigma / x)$; in Case II equality holds for the slit mapping defined by $\alpha(x)=0$ in $b \leqslant x \leqslant \sigma$ and $(x)=\cos ^{-1}(\sigma / x)$ in $\sigma<x<1$. The complete description of all extremal functions can be found in [1] and [3] for the respective inequalities (1) and (2).

\section{REFERENCES}

1. D. W. DeTemple, Generalizations of the Grunsky-Neharn inequalities, Arch. Rational Mech. Anal. 44 (1971), 93-120.

2. W. K. Hayman, Multivalent functions, Cambridge Univ. Press, London and New York, 1958.

3. J. A. Jenkins, On certain coefficients of univalent functions, Analytic Functions, Princeton Univ. Press, Princeton, N.J., 1960, pp. 159-194.

4. G. B. Leeman, Jr., $A$ new proof for an inequality of Jenkins, Proc. Amer. Math. Soc. 54 (1976), $114-116$.

Department of Pure and Applied Mathematics, Washington State University, PullMAN, WASHINGTON 99164

Department of Mathematics, Washington University, St. Louis, Missouri 63130 